\title{
Gender Identity Disorders and Multiple Sclerosis Risk: A National Record-Linkage Study
}

Julia Pakpoor, Clare J Wotton, Raph Goldacre, Klaus Schmierer, Gavin Giovannoni, Michael Goldacre

${ }^{1}$ Unit of Health-Care Epidemiology, Nuffield Department of Population Health, University of Oxford, Oxford, United Kingdom; ${ }^{2}$ Blizard Institute, Queen Mary University of London, Barts and the London School of Medicine and Dentistry, London, United Kingdom; ${ }^{3}$ Barts Health NHS Trust, The Royal London Hospital, Department of Neurology, London UK

Background: The demographic profile of multiple sclerosis (MS) implicates gender as an important factor in influencing disease risk and clinical course. Sex hormones may be important in mediating gender differences in MS, but their influence on MS risk remains largely uncharacterised. We hypothesize that an altered balance of sex hormones in males with gender identity disorders (GID) will both inherently, and secondary to treatment in undergoing male-to-female conversion (which typically involves taking feminizing hormones, anti-androgens and/or sex reassignment surgery), increase MS risk.

Objective: To investigate a potential association between GID and subsequent MS risk. Methods: We analysed linked English national Hospital Episode Statistics from 1999 to 2011. A cohort of males with GID and a cohort of females with GID were constructed by identifying the first episode of day-case care or hospital admission in which a GID or sexual transformation procedure was coded (using the International Classification of Diseases and the Office of Population Censuses and Surveys Classification of Interventions and Procedures). A reference cohort was similarly constructed from individuals admitted for various other minor medical and surgical conditions. We searched for any subsequent daycase care or inpatient admission for, or death from, MS in these cohorts. We calculated 
rates for MS, stratified and then standardized by age, calendar year of first recorded admission, region of residence, and socio-economic status.

Results: There were 1157 males and 2390 females in the GID cohorts, and 4.6 million males and 3.4 million females in the respective reference cohorts. The adjusted rate ratio (RR) of MS following GID in males was 6.63 (95\% confidence interval $(95 \% \mathrm{Cl}) 1.81-17.01, p=0.0002)$, based on 4 observed cases and 0.6 expected. The adjusted RR of MS following GID in females was $1.44(95 \% \mathrm{Cl}(0.47-3.37), \mathrm{p}=0.58)$, based on 5 observed cases and 3.5 expected. Conclusions: We report a strong positive association (a near seven-fold elevation of rates) between GID and subsequent MS in males. A strength of this study is the use of a huge dataset to study an otherwise extremely difficult logistical undertaking. Our findings support a previously reported association between testicular hypofunction and MS risk, and a potential role for low testosterone as a MS risk factor in males. We highlight a need for further exploration of the influence of feminizing sex hormones on MS risk. 